\title{
Non-linear Frequency Scaling Algorithm for FMCW SAR Data
}

\author{
Adriano Meta*, Peter Hoogeboom ${ }^{\dagger *}$ and Leo P. Ligthart* \\ *International Research Centre for Telecommunications and Radar, \\ Delft University of Technology, The Netherlands, Email: a.meta@irctr.tudelft.nl \\ ${ }^{\dagger}$ TNO Defence, Security and Safety, The Hague, The Netherlands
}

\begin{abstract}
This paper presents a novel approach for processing data acquired with Frequency Modulated Continuous Wave (FMCW) dechirp-on-receive systems by using a non-linear frequency scaling algorithm. The range frequency non-linearity correction, the Doppler shift induced by the continuous motion and the range migration removal are performed at the same time in the wavenumber domain with a very efficient solution. The cross-range focusing is then achieved with conventional matched filtering. Real data have been acquired with the FMCW SAR demonstrator system built at the Delft University of Technology. Stripmap images processed with the proposed method are presented in order to show the validity of the non-linear frequency scaling algorithm.
\end{abstract}

Index terms - Synthetic Aperture Radar (SAR), Frequency Modulated Continuous Wave (FMCW), range frequency nonlinearity, frequency scaling algorithm.

\section{INTRODUCTION}

The combination of Frequency Modulated Continuous Wave (FMCW) technology and Synthetic Aperture Radar (SAR) techniques leads to lightweight, cost effective, low power consuming imaging sensors of high resolution [1] [2]. FMCW sensors can continuously transmit linear chirps, and the use of dechirp-on-receive configurations allows an high reduction of the sampling frequency. However, the presence of nonlinearities in the transmitted signal is limiting the use of high resolution FMCW systems based on simple VCO technology to short range applications. In fact, non-linearities deteriorate the range resolution when deramping techniques are used because they spread the target energy through different frequencies [3]. Algorithms which remove such a limitation can allow the use of very cheap system, still reaching nearly optimal performances. Very recently, a method which removes the effects of frequency non-linearities in deramped FMCW data has been published [4].

For the processing of SAR data, several algorithms have been developed and each of them has different characteristics which make them appropriate for different applications. Particularly, the frequency scaling algorithm [5] is able to correct for the range migration processing deramped data and without using interpolations. FMCW SAR data, however, differ from conventional pulse SAR data also for the presence of an additional linear range migration, induced by the continuous motion of the aircraft while transmitting and receiving the signals [6]. That means, the stop-and-go approximation, used in the development of conventional SAR algorithms, could not hold anymore.

The novel approach presented in the paper aims to combine the non-linearity correction and the frequency scaling algorithms and the removal of the FMCW Doppler shift. The two methods are based on residual video phase removal, therefore this fact can be exploited to integrate the algorithms and decrease the, already low, computational load. The result is the non-linear frequency scaling algorithm, which is presented in this paper. It has a computational load nearly equal to the conventional method, with the advantage of additionally correcting the Doppler shift effect induced by the continuous motion and the frequency non-linearity degradation. Therefore, the proposed algorithm is very suitable for the processing of FMCW SAR data.

The remaining of the paper is structured in four sections. Section II introduces the deramped FMCW signal taking into account the frequency non-linearity and without using the stop-and-go approximation. Successively, the combination of the FMCW Doppler shift, frequency non-linearity and range migration correction is described in section III. Section IV provides results of the developed non-linear frequency scaling algorithm applied to real data. The data set has been collected with the FMCW SAR demonstrator system developed at the Delft University of Technology. Finally, conclusions are stated in section $\mathrm{V}$.

\section{DERAMPED NON-LINEAR FMCW SAR SIGNAL}

This section derives an analytical development of the deramped FMCW SAR signal in the two-dimensional frequency domain including the frequency non-linearity and without using the stop-and-go approximation. The radar continuously transmits linear FM chirps having duration $T_{p}$ equal to the pulse repetition interval $P R I$, chirp rate $\alpha=B / P R I$, where $B$ is the transmitted bandwidth and center frequency $f_{c}$. The transmitted frequency is expressed as:

$$
f_{r}=f_{c}+\alpha t+e(t)
$$

where $-P R I / 2 \leq t<P R I / 2$ and $e(t)$ is the frequency nonlinearity term, which is unwanted. In dechirp-on-receive systems, the received and transmitted signal are mixed in order 

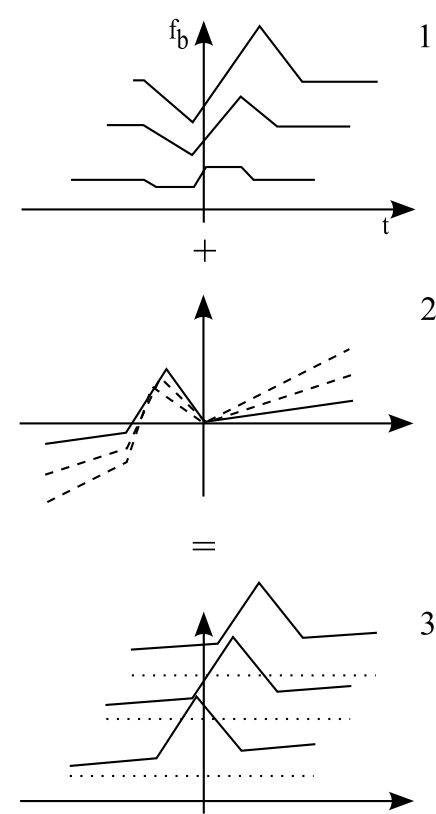

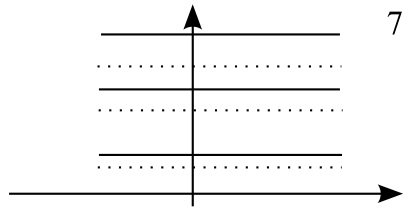

Non-linear

Frequency

Scaling

Algorithm

6

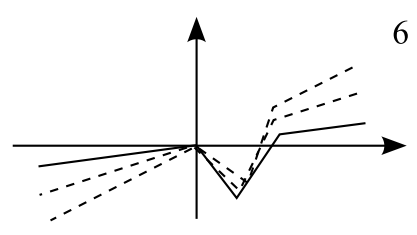

4

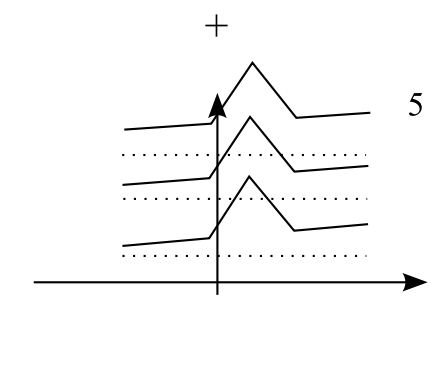

Fig. 1. Schematic representation of the non-linear frequency scaling algorithm. 1) raw data of three point scatterers. 2) Frequency scaling, Doppler shift, and transmitted non-linearity reference function. 3) Signal data after the multiply with the first reference function. 4) Residual video phase correction function. 5) Signal data after residual video phase correction. 6) Removal of the spreading induced by the frequency scaling and by the received non-linearity. 7) Signal after complete non-linear frequency scaling. Range time is on the horizontal axis whereas vertical axes represent range frequency. In 2) and 6) dashed curves indicate the dependency on the Doppler frequency.

to reduce the required system sampling rate. In a stripmap configuration with the radar flying along a straight path, the target time delay is:

$$
\tau=\frac{2}{c} \sqrt{R^{2}+v^{2}(t+T)^{2}}
$$

where $R$ is the distance of closest approach, $v$ is the aircraft velocity and $T$ is the slow time variable. The deramped FMCW SAR signal can be described by:

$$
\begin{aligned}
s(t, T)= & \exp \left(-j 2 \pi\left(f_{c} \tau+\alpha \tau t\right.\right. \\
& \left.\left.-\frac{1}{2} \alpha \tau^{2}+e(t)-e(t-\tau)\right)\right)
\end{aligned}
$$

The first exponential term is the azimuth phase history; the second term is the range signal while the third term is called Residual Video Phase (RVP) [7]. The last two terms of (3) represent the unwanted contribution of the frequency non-linearity; $e(t)$ can be directly measured or estimated from the raw data [4] and is assumed known in the rest of the analysis. Frequency non-linearities seriously degrade the system performances if not corrected for. Applying a Fourier transform with respect to the variable $T$ to (3), and after developing the calculations, the signal in the range-Doppler domain can be expressed as:

$$
\begin{aligned}
s\left(t, f_{D}\right)= & \left(\exp \left[-j \frac{4 \pi R \beta\left(f_{D}\right)}{\lambda}\right] \exp \left[-j \frac{4 \pi \alpha R}{c \beta\left(f_{D}\right)} t\right]\right. \\
& \exp \left(j 2 \pi f_{D} t\right) \exp \left[j\left(e(t)-e\left(t-\frac{2 R}{c \beta\left(f_{D}\right)}\right)\right)\right] \\
& \left.\operatorname{src}\left(t, f_{D} ; R\right)\right) * \exp \left(-j \pi \alpha t^{2}\right)
\end{aligned}
$$

Differently from [5], the expression in (4) includes the nonlinearity and the Doppler shift terms The Doppler frequency $f_{D}$ varies within:

$$
\frac{-P R F}{2}+f_{D c} \leq f_{D} \leq \frac{P R F}{2}+f_{D c}
$$

where $P R F$ is the pulse repetition frequency and $f_{D c}$ is the Doppler centroid. The first two terms of (4) represent the azimuth phase history and the range modulation. The factor $\beta\left(f_{D}\right)$ describes the range cell migration (RCM) as a function of the Doppler frequency:

$$
\beta\left(f_{D}\right)=\sqrt{1-\frac{f_{D}^{2} \lambda^{2}}{4 v^{2}}}
$$

The third and fourth terms are the consequences of the continuous motion within the sweep, which introduces a Doppler shift, and of the presence of range frequency non-linearity. The 
modified non-linear frequency scaling algorithm differs from its conventional method for the ability to remove their effects.

The $\operatorname{src}\left(t, f_{D} ; R\right)$ term in (4) is the secondary range compression and its expression can be found in [5], for instance.

\section{FREQUENCY NON-LINEARITY, DOPPLER SHIFT AND RANGe Cell Migration CORRECTION}

The non-linearity and RCM removal are obtained through the exploitation of the residual video phase. The two corrections are achieved at the same time. In fact, the frequency scaling can be thought as a special case of frequency nonlinearity correction, namely a linear case. Additionally, also the Doppler shift effects, induced by the continuous motion within the sweep [6] [8] [9], can be corrected at this stage.

The first step is a multiply of (4) with the following reference function:

$$
H_{1}\left(t, f_{D}\right)=\exp \left[-j\left(2 \pi f_{D} t+e(t)-\pi \alpha t^{2}\left(1-\beta\left(f_{D}\right)\right)\right)\right]
$$

which removes the Doppler shift, the transmitted non-linearity and scales the frequency. Successively, the signal is Fourier transformed with respect to $t$ and multiplied with the RVP correction term:

$$
H_{2}\left(f, f_{D}\right)=\exp \left[-j \frac{\pi f^{2}}{\alpha \beta\left(f_{D}\right)}\right]
$$

An inverse Fourier transform brings the signal back to the time domain:

$$
\begin{aligned}
s\left(t, f_{D}\right)= & \exp \left[-j \frac{4 \pi R \beta\left(f_{D}\right)}{\lambda}\right] \exp \left[-j \frac{4 \pi \alpha R}{c} t\right] \\
& \exp \left[-j \pi \alpha t^{2}\left(\beta\left(f_{D}\right)^{2}-\beta\left(f_{D}\right)\right)\right] \\
& \exp \left[-j e_{R V P}(t)\right] \operatorname{src}\left(t, f_{D} ; R\right)
\end{aligned}
$$

where $e_{R V P}$ represents the non-linearity after it passed through the RVP filter. Finally a last reference function

$$
H_{3}\left(t, f_{D}\right)=\left(\exp \left[-j \pi \alpha t^{2}\left(\beta\left(f_{D}\right)^{2}-\beta\left(f_{D}\right)\right)\right]\right.
$$

multiplies (9) removing the, now equalized, spreading of the peak response induced by the frequency scaling and by the non-linearity. After this last step, the following expression describes the resulting signal:

$$
\begin{aligned}
s\left(t, f_{D}\right)= & \exp \left[-j \frac{4 \pi R \beta\left(f_{D}\right)}{\lambda}\right] \exp \left[-j \frac{4 \pi \alpha R}{c} t\right] \\
& \operatorname{src}\left(t, f_{D} ; R\right)
\end{aligned}
$$

At this point, conventional steps can be performed to achieve secondary range compression and successively azimuth focusing. The proposed modified non-linear frequency scaling algorithm is very suitable for the processing of FMCW SAR data. In fact, it corrects, with practically no additional computational cost when compared with the conventional frequency scaling algorithm, the Doppler shift induced by the continuous motion in FMCW SAR and, particularly important, the degradation caused by the frequency non-linearity. This last characteristic, in fact, allows the use of very simple and cheap sensors which can generate the transmitted modulation directly in the radio frequency band, [1]. A schematic representation of the algorithm is illustrated in fig 1 .

\section{REAL DATA VALIDATION}

A demonstrator system has been realized at the International Research Centre for Telecommunication and Radar (IRCTR) of the Delft University of Technology, The Netherlands, using a $10 \mathrm{GHz}$ FMCW front-end. In the autumn of 2005, a very successful flight test campaign was carried out in Strausberg, Germany, and images were produced, demonstrating the great potential of such a system [1]. Some collected data have been processed with the method developed in the paper to verify its validity. Figure 2(a) shows an FMCW SAR image obtained applying conventional frequency scaling algorithm, while fig. 2(b) has been produced with the aim of the proposed non-linear frequency scaling algorithm. For this images, the system was transmitting a bandwidth of $260 \mathrm{MHz}$ and $P R I$ of $1 \mathrm{~ms}$, centered around a carrier frequency of $9.6 \mathrm{GHz}$. Hamming windowing has been used both in range and azimuth focusing. For the cross-range compression a Doppler bandwidth of $200 \mathrm{~Hz}$ is processed; successively, spatial multilooking with a factor of 8 is performed. The pictures show an urban area close to the Strausberg airport. The improvements due to the range non-linearities correction are evident, both in terms of resolution and contrast, which is a direct consequence of an improved peak to integrated sidelobe ratio.

A similar comparison is reported in fig. 3(a) and fig. 3(b); the FMCW SAR image reproduced has been collected over a forest area with a low resolution configuration with a transmitted bandwidth of $130 \mathrm{MHz}$ and PRI of $0.5 \mathrm{~ms}$.

\section{CONCLUSION AND FUTURE WORK}

This paper has presented a modified non-linear frequency scaling algorithm for processing FMCW SAR data acquired with dechirp-on-receive systems. The range frequency nonlinearity correction, the Doppler shift induced by the continuous motion and the range migration removal are performed at the same time in the wavenumber domain with a very efficient solution. The computational load of the described non-linear frequency scaling algorithm is practically the same as the conventional one. Therefore, the proposed method is very suitable for the processing of FMCW SAR data. The cross-range focusing is then performed with conventional matched filtering. Two stripmap images, which have been acquired with the FMCW SAR demonstrator system built at the Delft University of Technology, have been presented to show the validity of the proposed non-linear frequency scaling algorithm. A qualitatively analysis indicates drastic improvements. Future work includes a complete quantitative description.

\section{ACKNOWLEDGEMENT}

This work is supported by the Technology Foundation STW, applied science division NWO and the technology program of the Dutch Ministry of Economic Affairs and part of it is being patented under the European Patent application No. 06076057.6. The project is carried out in close cooperation 


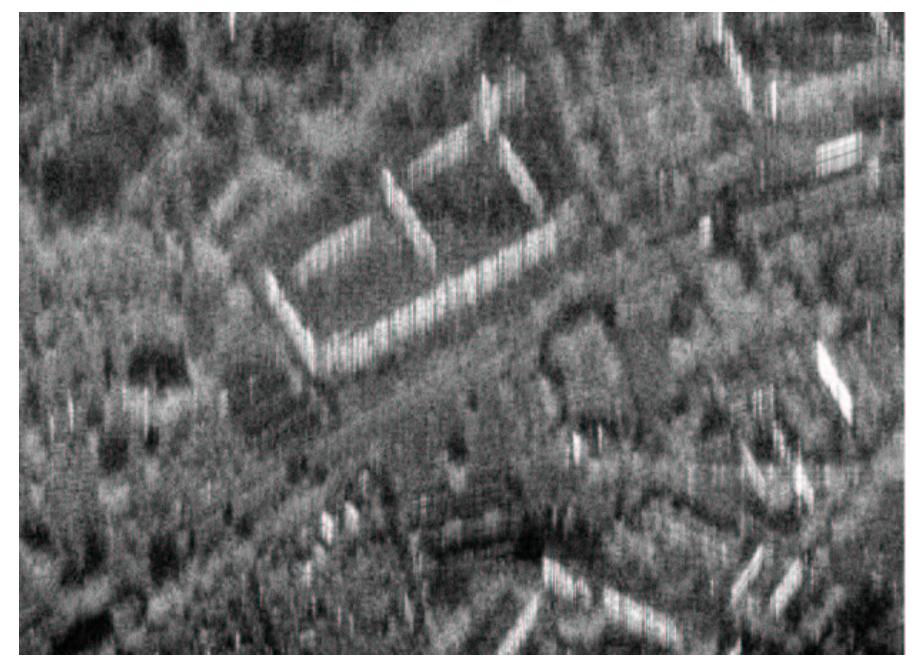

(a)

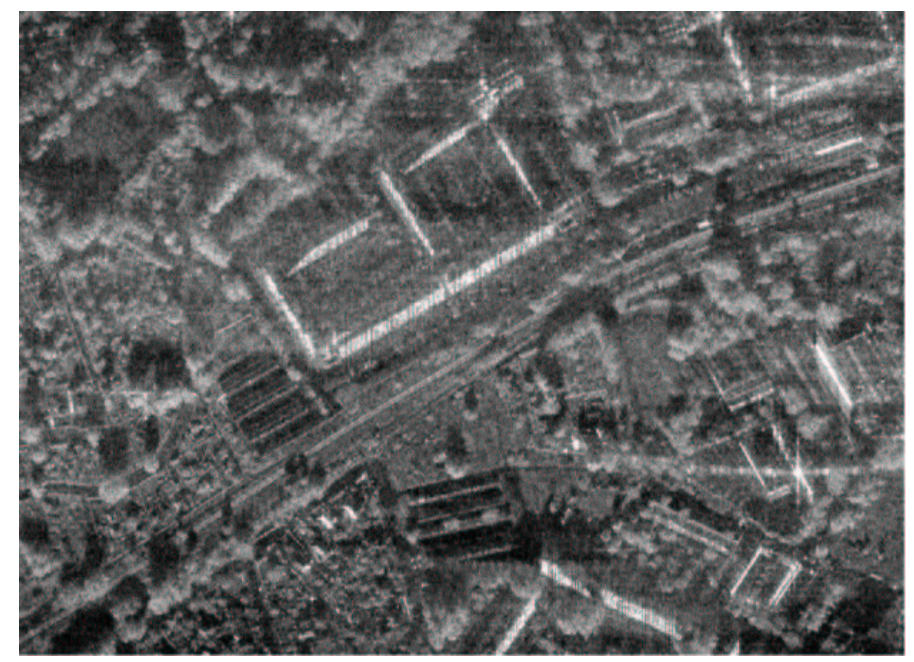

(b)

Fig. 2. FMCW SAR image obtained processing the raw data with conventional frequency scaling algorithm (a) and with the proposed non-linear version (b). Slant range is on the vertical axis. The image has been acquired over a urban area transmitting $260 \mathrm{MHz}$ bandwidth

with the TNO Defence, Security and Safety in The Hague, The Netherlands, and Eonic in Delft, The Netherlands.

\section{REFERENCES}

[1] A. Meta, P. Hakkaart, F. V. der Zwan, P. Hoogeboom, and L. Ligthart, "First demonstration of an X-band airborne FMCW SAR," in Proc. EUSAR'06, Dresden, Germany, May 2006.

[2] M. Edrich, "Design Overview and flight Test Results of the Miniaturised SAR Sensor MISAR," in Proc. EuRAD'04, Amsterdam, The Netherlands, Oct. 2004, pp. 205-208.

[3] S. O. Piper, "Homodyne FM-CW Radar Resolution Effects with Sinusoidal Nonlinearities in the Frequency Sweep," in Proc. IEEE Int. Radar Conf. '95, Alexandria, USA, May 1995, pp. 563-567.

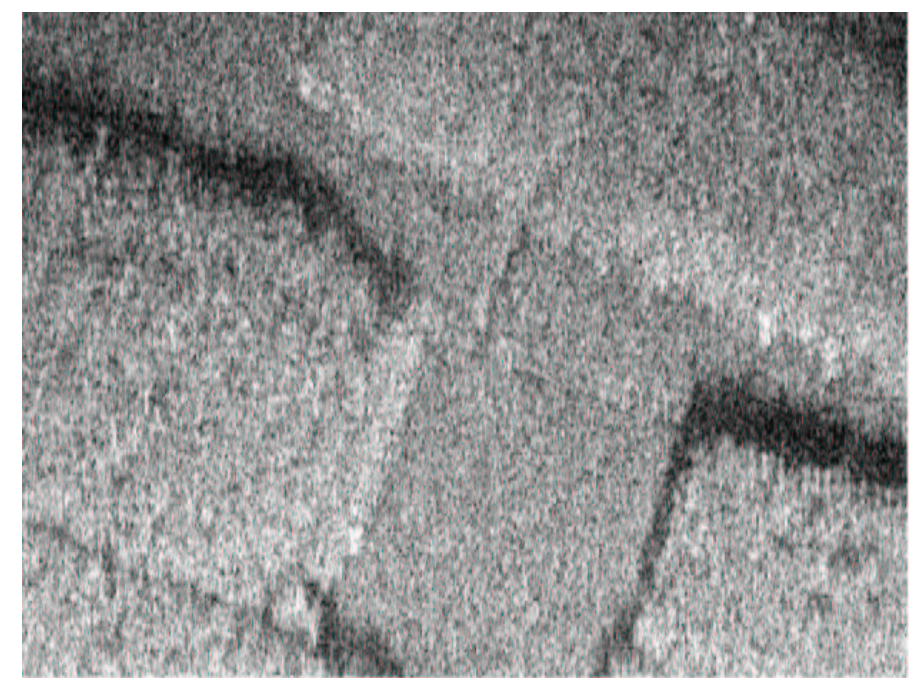

(a)

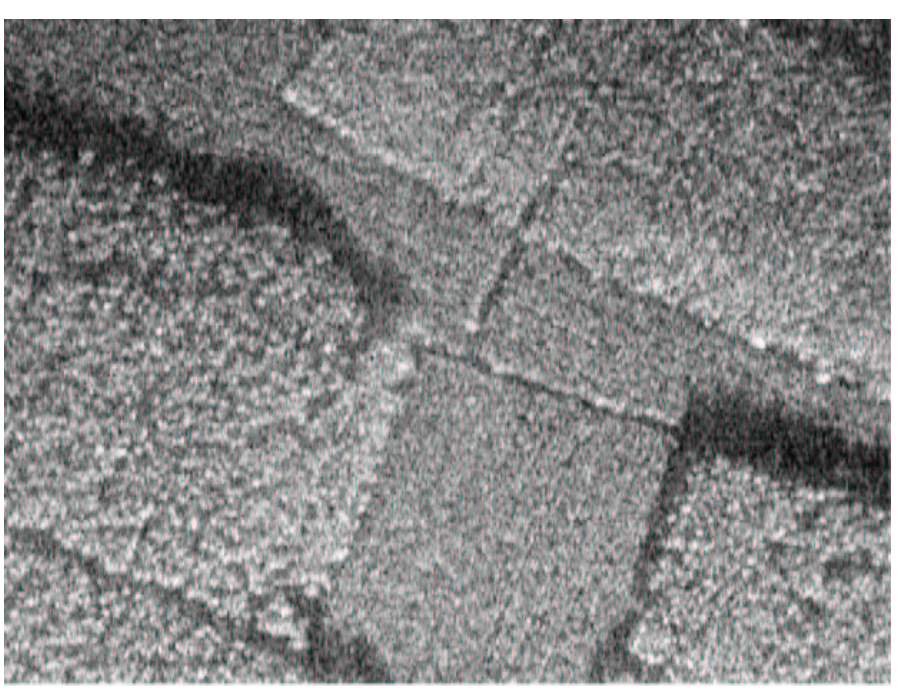

(b)

Fig. 3. The same as figure 2. The image has been acquired over a vegetation area transmitting $130 \mathrm{MHz}$ bandwidth

[4] A. Meta, P. Hoogeboom, and L. Ligthart, "Range non-linearities correction in FMCW SAR," in Proc. IGARSS'06, Denver, USA, July 2006, p. in Press.

[5] J. Mittermayer, A. Moreira, and O. Loffeld, "Spotlight SAR data processing using the Frequency Scaling algorithm," IEEE Trans. on Geosci. and Remote Sensing, vol. 37, no. 5, pp. 2198-2214, Sep. 1999.

[6] A. Meta and P. Hoogeboom, "Signal Processing Algorithms for FMCW Moving Target Indicator Synthetic Aperture Radar,' in Proc. IGARSS'05, Seoul, Korea, July 2005, pp. 316-319.

[7] W. G. Carrara, R. S. Goodman, and R. M. Majewski, Spotlight Synthetic Aperture Radar. Boston, Artech House Inc., 1995.

[8] A. Meta, P. Hoogebbom, and L. Ligthart, "Correction of the effects induced by the continuous motion in FMCW SAR," in Proc. IEEE Radar Conf. '06, Verona, NY, USA, Apr. 2006.

[9] J. J. M. de Wit, A. Meta, and P. Hoogeboom, "Modified range-Doppler processing for FM-CW synthetic aperture radar," IEEE Geosci. Remote Sensing Letters, vol. 3, no. 1, pp. 83-87, Jan. 2006. 